\begin{tabular}{ccc}
\hline & Journal of Advanced Computer Science \& Technology, 9(1) (2020) 11-13 \\
SPC & Website: $w$ ww.sciencepubco.com/index.php/JACST \\
Research paper & Technology \\
\hline
\end{tabular}

\title{
Geometric Approach to Optimal Path Problem with Uncertain Arc Lengths
}

\author{
Atefeh Hasan-Zadeh ${ }^{1 *}$ \\ ${ }^{1}$ Fouman Faculty of Engineering, College of Engineering, University of Tehran, Iran \\ *Corresponding author E-mail:hasanzadeh.a@ut.ac.ir
}

\begin{abstract}
In this paper, the problem of finding the shortest paths, one of the most important problems in science and technology has been geometrically studied. Shortest path algorithm has been generalized to the shortest cycles in each homotopy class on a surface with arbitrary topology, using the universal covering space notion in the algebraic topology. Then, a general algorithm has been presented to compute the shortest cycles (geometrically rather than combinatorial) in each homotopy class. The algorithm can handle surface meshes with the desired topology, with or without boundary. It also provides a fundamental framework for other algorithms based on universal coverage space due to the capacity and flexibility of the framework.
\end{abstract}

Keywords: Shortest path problem; Homotopy; Covering space; Cycle

\section{Introduction}

The shortest problem is finding a path with minimal distance, time, or cost between the source node and the destination node. Many programming problems, including transportation, routing, communications, and supply chain management, can be seen as special cases of the shortest path problem. In the 1950s and 1960s, effective algorithms were proposed or developed by Bellman [1], Dijkstra [2], Dreyfus [3] and Floyd [4], which made the short-haul problem a central element of a network.

These algorithms have been called classical algorithms. In classical algorithms, the network must have deterministic arc lengths. However, for reasons of failure, maintenance or other reasons, arc lengths are not deterministic in many situations. Therefore, it is inappropriate to use conventional algorithms in these situations. Some researchers have estimated that these non-deterministic phenomena are compatible with randomness and have introduced the theory of probabilities into the shortest path problem; see Frank [5], Hall [6], Loui [7], Mirchandani [8], for example. Since Dubois and Prade [9] proposed the Short Fuzzy Path (FSPP) problem in 1980, the fuzzy theory has begun to attract networked researchers. Some researchers, such as Klein [10], Ji and Iwamura [11] have worked extensively in this area.

In 2007, Liu [12] proposed the theory of uncertainty to describe non-deterministic phenomena, especially expert data and subjective estimates. Since then, the theory of uncertainty has provided a new approach to addressing non-deterministic factors in programming problems. Obviously, the length of each path in an uncertain network is uncertain and we cannot get the shortest path in the normal direction. What we are studying is the distribution of the length of the shortest path. Nowadays, the concepts of the time-dependent shortest paths in large graphs and the shortest path in an uncertain network is taken into consideration. These two concepts are duels of each other and constitute the best path under certain constraints of the level of confidence [13], [14-15]. However, the exact methods for finding their shortest path is not indicated.

Solving problems with routes with a geometric approach will lead to useful extensions of them [16]. In this paper, after a literature review about the shortest path problem algorithms, a new approach has been presented. In the sequel, algebraic concepts has been explained and then, the shortest path problem can be solved geometrically rather than combinatorial on a surface with arbitrary topology with or without boundary.

\section{Literature Review}

Shortest path problem is finding a path between two vertices (or nodes) of a graph so as to minimize the sum of the weights of its constituent edges.

The most important algorithms to solve this problem are [17-18]:

Dijkstra's algorithm solves the problem of the shortest single source path.

The Bellman-Ford algorithm solves the single source problem if the edge weights can be negative.

A *-search algorithm solves the shortest path of a pair using heuristic methods to try to speed up the search.

The Floyd-Warshall algorithm solves all the shortest paths in pairs. 
Johnson's algorithm solves all the shortest path pairs and can be faster than Floyd-Warshall on sparse graphs.

The Viterbi algorithm solves the shortest stochastic path problem with additional probabilistic weight on each node.

The problem of finding the shortest paths in graphs is a fundamental optimization problem for many applications, such as routing in networks, image segmentation in vision, surface segmentation in graphics, motion and robot navigation, speech recognition and VLSI design, etc.

\section{Methodology}

The concepts presented in this section can be found in the standard books of geometry and algebraic topology, including [19]. A continuous map $f: I \rightarrow X$ where $I$ is the unit interval $[0,1]$ and $X$ is a space is called a path in $X$. The idea of permanently deforming a path while keeping its determined ends is specified by the following definition. A homotopy of paths in $X$ is a family $f_{t}: I \rightarrow X, 0 \leq t \leq 1$, such that

(1) The extremities $f_{t}(0)=x_{0}$ and $f_{t}(1)=x_{1}$ are independent of $t$.

(2) The associated map $F: I \times I \rightarrow X$ defined by $F(s, t)=f_{t}(s)$ is continuous. When two paths $f_{0}$ and $f_{1}$ are connected in this way by a homotopy $f_{t}$, they are called homotopes. The notation for this is $f_{0} \square f_{1}$.

Let $f, g: I \rightarrow X$ be such that $f(1)=g(0)$, there exists a path of composition or of product $f . g$ which crosses first $f$ then $g$, defined by the formula

$$
f . g(s)=\left\{\begin{array}{cc}
f(2 s) & 0 \leq s \leq \frac{1}{2} \\
g(2 s-1) & \frac{1}{2} \leq s \leq 1
\end{array}\right.
$$

Thus, $f$ and $g$ are traveled twice as fast so that $f . g$ is traveled in the unit time. This produces operation respects the homotopy classes because if $f_{0} \square f_{1}$ and $g_{0} \square g_{1}$ via homotopies $f_{t}$ and $g_{t}$, and if $f_{0}(1)=g_{0}(0)$ so that $f_{0} \cdot g_{0}$ is defined, then $f_{t}$. $g_{t}$ is defined and provides a homotopy $f_{0} \cdot g_{0} \square f_{1} \cdot g_{1}$.

In particular, the main focus will be on the paths $f: I \rightarrow X$ with the same starting and ending points $f(0)=f(1)=x_{0} \in X$. These paths are called loops and the point of common start and end $x_{0}$ is called the base point. The set of all the homotopy classes $[f]$ of the loops $f: I \rightarrow X$ at the base point $x_{0}$ is denoted $\pi_{1}\left(X, x_{0}\right)$.

$\pi_{1}\left(X, x_{0}\right)$ is a group with respect to the product $[f][g]=[f . g]$. This group is called the fundamental group of $X$ at the base point $x_{0}$. Thus, if $X$ is path-connected, the group $\pi_{1}\left(X, x_{0}\right)$ is, up to isomorphism, independent of the choice of base point $x_{0}$. In this case, the notation $\pi_{1}\left(X, x_{0}\right)$ is often abbreviated to $\pi_{1}(X)$, or $\pi_{1} X$.

In general, a space is called simply connected if it is path-connected and has a trivial fundamental group.

A space $X$ is simply connected if and only if there is a unique homotopy class of paths connecting any two points of $X$.

Let $X$ be a space, a covering space of $X$ consists of a space $\tilde{X}$ and a map $p: \tilde{X} \rightarrow X$ satisfying the following condition:

For each point $x \in X$, there exists an open neighborhood $U$ of $x$ in $X$ such that $p^{-1}(U)$ is a union of disjoint open sets, each of which is mapped homeomorphically onto $U$ by $p$.

\section{Results}

Theorem. The shortest path problem can be solved geometrically rather than combinatorial on a surface with arbitrary topology with or without boundary.

Proof. As mentioned about homotopy paths, without losing the whole, it can be studied a restricted version of the above problem:

Restricted shortest cycle problem: With a surface mesh $X$ with an arbitrary topology, it should be found in each homotopy class the shortest cycle passing through a given point $x_{0} \in X$.

For this purpose, it has been proposed a general framework using universal covering space to turn the problem from the search of the shortest cycles into a problem of finding the shortest paths. In order to avoid the exponential blowing up of the space required by naïve when building the universal covering space, it suffices a space efficient data structure to manage the universal covering space will be developed.

On the other hand, there are three facts about coverage spaces $p: \tilde{X} \rightarrow X$.

(a) For each path $f: I \rightarrow X$ starting at a point $x_{0} \in X$ and each $\tilde{x}_{0} \in p^{-1}\left(x_{0}\right)$, there exists a unique lift $\tilde{f}: I \rightarrow \tilde{X}$ starting at $\tilde{x}_{0}$.

(b) For each homotopy $f_{t}: I \rightarrow X$ of paths beginning with $x_{0}$ and each $\tilde{x}_{0} \in p^{-1}\left(x_{0}\right)$, there exists a unique high homotopy $\tilde{f}_{t}: I \rightarrow \tilde{X}$ of paths starting with $\tilde{x}_{0}$.

(c) Let map $F: Y \times I \rightarrow X$ and map $\tilde{F}: Y \times\{0\} \rightarrow \tilde{X}$ by raising $\left.F\right|_{Y \times\{0\}}$, there exists then a unique map $\tilde{F}: Y \times I \rightarrow \tilde{X}$ raises $F$ and limits to $\tilde{F}$ given on $Y \times\{0\}$.

In the sequel of the process, a set of homology bases as a section graph by cutting will be used, the section graph in the original mesh will become the cutting limit in the fundamental domain.

In this way, finding the shortest path is limited to calculate a fundamental domain $\tilde{X}$ by cutting the mesh $X$ along a set of curves. Then by taking a central copy of $\tilde{X}$ and glue more copies of it along the cut segments, the optimal path is found. 


\section{Conclusions}

A general algorithm had been presented to compute the shortest cycles (geometrically rather than combinatorial) in each homotopy class. It is proved that the shortest path problem can be solved geometrically rather than combinatorial on a surface with arbitrary topology with or without boundary.

\section{References}

[1] Bellman E, (1958), On a routing problem, Quarterly of Applied Mathematics, 16(1), 87-90.

[2] Dijkstra EW, (1959), A note on two problems in connection with graphs, Numerical Mathematics, 1(1), $269-271$.

[3] Dreyfus S, (1969), An appraisal of some shortest path algorithms, Operations Research, 17(3), 395-412

[4] Floyd RW, (1962), Algorithm-97-shortest path, Communications of the ACM, 5(6), 345.

[5] Frank H, (1969), Shortest paths in probability graphs, Operations Research, 17(4), 583-599.

[6] Hall R, (1986), The fastest path through a network with random time-dependent travel time, Transportation Science, 20(3), 182-188.

[7] Loui P, (1983), Optimal paths in graphs with stochastic or multidimensional weights, Communications of the ACM, 26(9), 670-676.

[8] Mirchandani PB, (1976), Shortest distance and reliability of probabilistic networks, Computers \& Operations Research, 3(4), 347-676.

[9] Dubois D, Prade H, Fuzzy Sets and Systems: Theory and Applications, Academic Press, New York, 1980.

[10] Klein CM, Fuzzy shortest paths, Fuzzy Sets and Systems, 39(1), 27-41.

[11] Ji X \& Iwamura K, (2007), New models for shortest path problem with fuzzy arc lengths, Applied Mathematical Modelling, 31(2), 259-269.

[12] Baoding Liu, Uncertainty Theory, 2007, Springer

[13] Idri A, Oukarfi M, Boulmakul A, Zeitouni K \& Masri A, (2017), A new time-dependent shortest path algorithm for multimodal transportation network, Procedia Computer Science, 109C, 692-697.

[14] Shirdel G \& Rezapour H, (2017), Approximate solutions for time-varying shortest path problem, Communications in Combinatorics and Optimization, 2(2), 39-147.

[15] Yang Y, Li Z, Wang X \& Hu Q, (2019), Finding the Shortest Path with Vertex Constraint over Large Graphs, Complexity, https://doi.org/10.1155/2019/8728245

[16] Cormen TH, Leiserson CE, Rivest RL \& Stein C, Introduction to algorithms, Massachusetts Institute of technology, 2001.

[17] Baras JS, Theodorakopoulos G \& Walrand J, Path Problems in Networks, Morgan and Claypool, 2010.

[18] Hasan-Zadeh A, (2019), Geometric Modelling of the Thinning by Cell Complexes, Journal of Advanced Computer Science \& Technology, 8(2), 3839.

[19] Hatcher, Algebraic Topology, Cambridge University Press, 2001. 